\title{
Adam17, a Target of Mir-326, Promotes Emt-Induced Cells Invasion in Lung Adenocarcinoma
}

\author{
Ming Cai ${ }^{a}$ Zhiqiang Wang ${ }^{a}$ Jiru Zhang ${ }^{b}$ Huan Zhou ${ }^{c}$ Linfang Jin ${ }^{d}$ Ruizhen Baid $^{d}$ \\ Yuan Wenga
}

\begin{abstract}
aDepartment of Thoracic and Cardiovascular Surgery, ${ }^{b}$ Department of Anesthesiology, 'Department of Clinical Laboratory, dDepartment of Pathology, Affiliated Hospital of Jiangnan University, The Forth People's Hospital of Wuxi City, Wuxi, People's Republic of China
\end{abstract}

\section{Key Words}

ADAM17 • miR-326 • Invasion • Lung adenocarcinoma • EMT

\begin{abstract}
Background/Aims: A disintegrin and metalloprotease (ADAM) 17 has been reported to be implicated in cancer cells invasion. Nevertheless, its potential role in lung adenocarcinoma has not been addressed clearly. Methods: RT-PCR and Western blot were used to detect the expression of miR-326 and ADAM17 in lung adenocarcinoma samples $(n=73)$. miR-326 mimics and inhibitor were tansfected in human A549 and SPCA1 cell lines. The transwell assay was used to detect the cell invasive ability. The regulation mechanism was evaluated by luciferase reporter assay. The markers of (epithelial-to-mesenchymal transition) EMT were detected by using Western blot assay. Results: We found increased expression of ADAM17 in lung adenocarcinoma and cell lines. In vitro, up-regulation of ADAM17 promoted cells invasion, while silencing of ADAM17 inhibited cells invasion. Meanwhile, ADAM17 could affect the markers of EMT. Furthermore, we confirmed that ADAM17 is a target of miR-326, which is involved in EMT and cells invasion. Conclusions: These findings revealed that ADAM17, a target of miR-326, promoted EMT-induced cells invasion in lung adenocarcinoma.
\end{abstract}

Copyright (C) 2015 S. Karger AG, Basel

M. Cai and Z. Wang contributed equally to this work.

Yuan Weng

KARGER 125
Department of Thoracic and Cardiovascular Surgery, Affiliated Hospital of Jiangnan University, The Forth People's Hospital of Wuxi City, Wuxi, 214062 (PR China) Tel. +86510 88682124, Fax +8651085808820, E-Mail jsshp@hotmail.com 


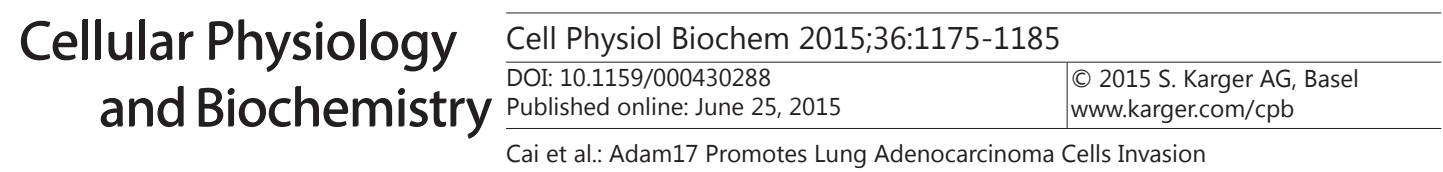

\section{Introduction}

Human lung cancer, one of the most common aggressive worldwide has the highest mortality rate among malignant tumors [1]. According to pathological pattern, nonsmall-cell lung cancer (NSCLC) is mainly divided into squamous cell carcinoma (SCC) and adenocarcinoma (AC). The overall 5-year survival rate for lung adenocarcinoma is extremely low. The prevalence of lung adenocarcinoma is rising, and lung adenocarcinoma is easy to metastasis. Thus, it is necessary to explore the mechanism of lung adenocarcinoma metastasis.

ADAM17 has a role in many biological processes of multiple tumors [2, 3]. In previous studies, ADAM17 is an important regulator of the tumorigenic properties of human NSCLC and may be used as a potential anticancer therapeutic target in NSCLC [4]. ADAM17 is overexpressed in non-small cell lung cancer and its expression correlates with poor patient survival [5]. However, the mechanism of ADAM17 in regulating lung adenocarcinoma cells invasion remains unclear.

microRNAs (miRNAs) play an crucial role in post-transcriptional regulation in the variety of cancer biological processes [6-9]. Recent studies showed that miR-326 functions as a tumor suppressor in colorectal cancer by targeting the nin one binding protein [10]. miR326 associates with biochemical markers of bone turnover in lung cancer bone metastasis [11]. Wang et al. reported that miR-326 is expressed abnormally between the non-small cell lung cancer metastatic and non-metastatic tissues, which provides experimental basis for exploring the mechanism of non-small cell lung cancer metastasis and provides a potential idea for molecular diagnosis and treatment [12].

In this study, we attempted to explore the underlying mechanism of ADAM17 and miR326 in the development of the lung adenocarcinoma.

\section{Material and Methods}

\section{Clinical Samples}

All the collected lung adenocarcinoma tissues and the corresponding adjacent samples from the 73 patients were stored at $-80^{\circ} \mathrm{C}$. The patients had undergone routine surgery from February 2007 and March 2010. This study was approved by the Ethical Committee of The Forth People's Hospital of Wuxi City.

\section{Cell culture}

The human lung adenocarcinoma cell lines (A549, H1299, SPCA1 and H1975) and normal lung cell (16HBE) were purchased from the Institute of Biochemistry and Cell Biology of the Chinese Academy of Sciences (Shanghai, China). All the cell lines were cultured in RPMI-1640 medium with 10\% fetal bovine serum (Invitrogen, Carlsbad, CA) and penicillin $(100 \mathrm{U} / \mathrm{ml})$ at $37^{\circ} \mathrm{C}$ with $5 \% \mathrm{CO}_{2}$.

\section{Isolation of total RNA and Quantitative RT-PCR}

Using TRIzol (Invitrogen, USA), RNA of all the tumor samples and corresponding adjacent tissues was extracted according to the manufacturer's instructions. Both miRNA and mRNA were reversely transcribed to cDNA. Then, relative ADAM17 mRNA expression level was examined by using SYBR Green quantitative real-time PCR (qRT-PCR). And GAPDH was used for normalization. The forward primer of ADAM17 was 5'ACT CTG AGG ACA GTT AAC CAA ACC-3' and the reverse primer was 5'- AGT AAA AGG AGC CAA TAC CAC AAG3'. miR-326 expression level was detected by using the TaqMan stem-loop qRT-PCR method with a mirVana miRNA Detection Kit and gene-specific primers, and normalized to U6. qRT-PCR assay was performed by using the ABI 7900 Fast Real-Time PCR system (ABI, CA, USA).

\section{Invasion assay}

Cells invasion assay was conducted with BioCoat Matrigel (BD Biosciences, San Jose, CA) and invasion chambers (Millipore, Eschborn, Germany) with an 8- $\mu$ m pore size according to the manufacturer's instructions. The chamber inserts were coated with $200 \mathrm{mg} / \mathrm{ml}$ BD Matrigel (BD Biosciences, San Jose, 


\section{Cellular Physiology Cell Physiol Biochem 2015;36:1175-1185

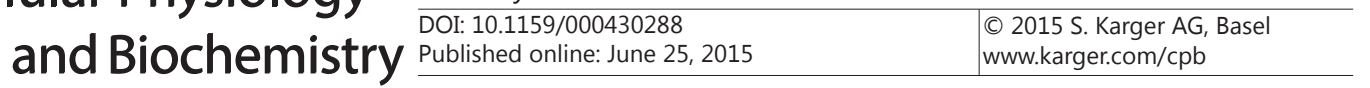 \\ Cai et al.: Adam17 Promotes Lung Adenocarcinoma Cells Invasion}

CA, USA). Cells $\left(5 \times 10^{4}\right)$ were incubated at $37^{\circ} \mathrm{C}$ for $24 \mathrm{~h}$, and then stained with crystal violet (Beyotime, Shanghai, China). A set of images was acquired by using NIS Elements image analysis software (Nikon, Tokyo, Japan). The values for cells invasion were obtained by counting three fields per membrane and represented the average of three independent experiments.

\section{Western blot assay}

The proteins were quantitated using a protein assay (bicinchoninic acid [BCA] method; Beyotime, Shanghai, China). And then the proteins were fractionated by sodium dodecyl sulfate polyacrylamide gel electrophoresis, transferred to polyvinylidene fluoride (PVDF) membrane, blocked in 4\% dry milk at room temperature for 1 hour, and immunostained with primary anti-ADAM17, anti-E-cadherin, anti-Ncadherin, anti-Vimentin (1:1000, Dizhao, Nanjing, China) and anti-GAPDH (1:5000, Kangchen, China). All results were visualized through a chemiluminescent detection system (Pierce ECL Substrate Western blot detection system, Thermo, Pittsburgh, PA) and then exposed in Molecular Imager ChemiDoc XRS System. The integrated density of the band was quantified by Image software (Bio-Rad, Hercules, CA).

\section{Plasmid construction and cell transfection}

The sequence of ADAM17 was synthesized (Jinweizhi, Suzhou, China). The plasmid was subcloned into lentiviral vector, and then co-transfected into HEK-293T cells with Lentiviral Packaging Mix, and followed by selection with G418. Cells were seeded into 6-well plates and transfected with 50 nM siRNA (small interfere RNA) targeting ADAM17 (siRNA/ADAM17: 5'- CAC AUG UAG AAA CAC UAC UTT-3') (GenePharma, Shanghai, China) using Lipofectamine 2000 (Invitrogen, USA) according to the instructions provided by the manufacturer.

Using Lipofectamine 2000 (Invitrogen, USA), A549 and SPCA1 cells were seeded into 6-well plates and transfected with $50 \mathrm{nM}$ of miR-326 mimics and miR-326 inhibitor along with NC (miR-control) and inhibitor NC (miR-326 inhibitor control), that were purchased from GenePharma (Shanghai, PR China). The transfection efficiency was monitored by using qRT-PCR. For the rescue experiment, A549 and SPCA1 cells were seeded into 6-well plates and transfected with siRNA/ADAM17 and siRNA/control (GenePharma, Shanghai, China) using Lipofectamine 2000 (Invitrogen, USA) at a final concentration of $100 \mathrm{nM}$.

\section{Luciferase reporter assay}

For the luciferase reporter assay, cells were cultured in 96 well plates and transfected with luciferase reporters (50 ng), and $50 \mathrm{nM}$ of (NC) miR-control, miR-326 mimics, pGL3-ADAM17 or pGL3-ADAM17-Mut (pGL3-ADAM17-Mutant). After 48 h, luciferase activity was measured using dual-luciferase reporter system (Promega, USA). The renilla activity was an internal control.

\section{Statistical methods}

The chi-squared test was used to test the significance of observed differences in the Table 1 data and the t-test was used for the other data analyses. All values are expressed as mean \pm SEM. Statistical analysis was performed using STAT11 and GraphPad Prism (version 5.01; GraphPad Software, Inc, La Jolla, CA) statistical software. $\mathrm{p}$ value $<0.05$ were considered significant.

\section{Results}

Elevated expression of ADAM17 promoted cells invasion in human lung adenocarcinoma

First, both in lung adenocarcinoma specimens $(n=73)$ as compared with corresponding adjacent tissues and lung adenocarcinoma cell lines (A549, H1299, SPCA1 and H1975) as compared with normal lung cell (16HBE), we detected the expression levels of ADAM17 by using qRT-PCR. Significantly, we discovered increased mRNA level of ADAM17 in tumor tissues ( $<<0.05$; Fig. 1A) and cell lines ( $<<0.05$; Fig. 1B). The aberrant expression of ADAM17 was highly associated with tumor stage and metastasis of patients (Table 1). Furthermore, to analysis the correlation between ADAM17 overexpression and lung adenocarcinoma metastasis, we compared ADAM17 expression in 10 pairs of $\mathrm{N}_{2}$ stage (LN metastasispositive) and $\mathrm{N}_{0}$ stage (LN metastasis negative) lung adenocarcinoma specimens. qRT-PCR 


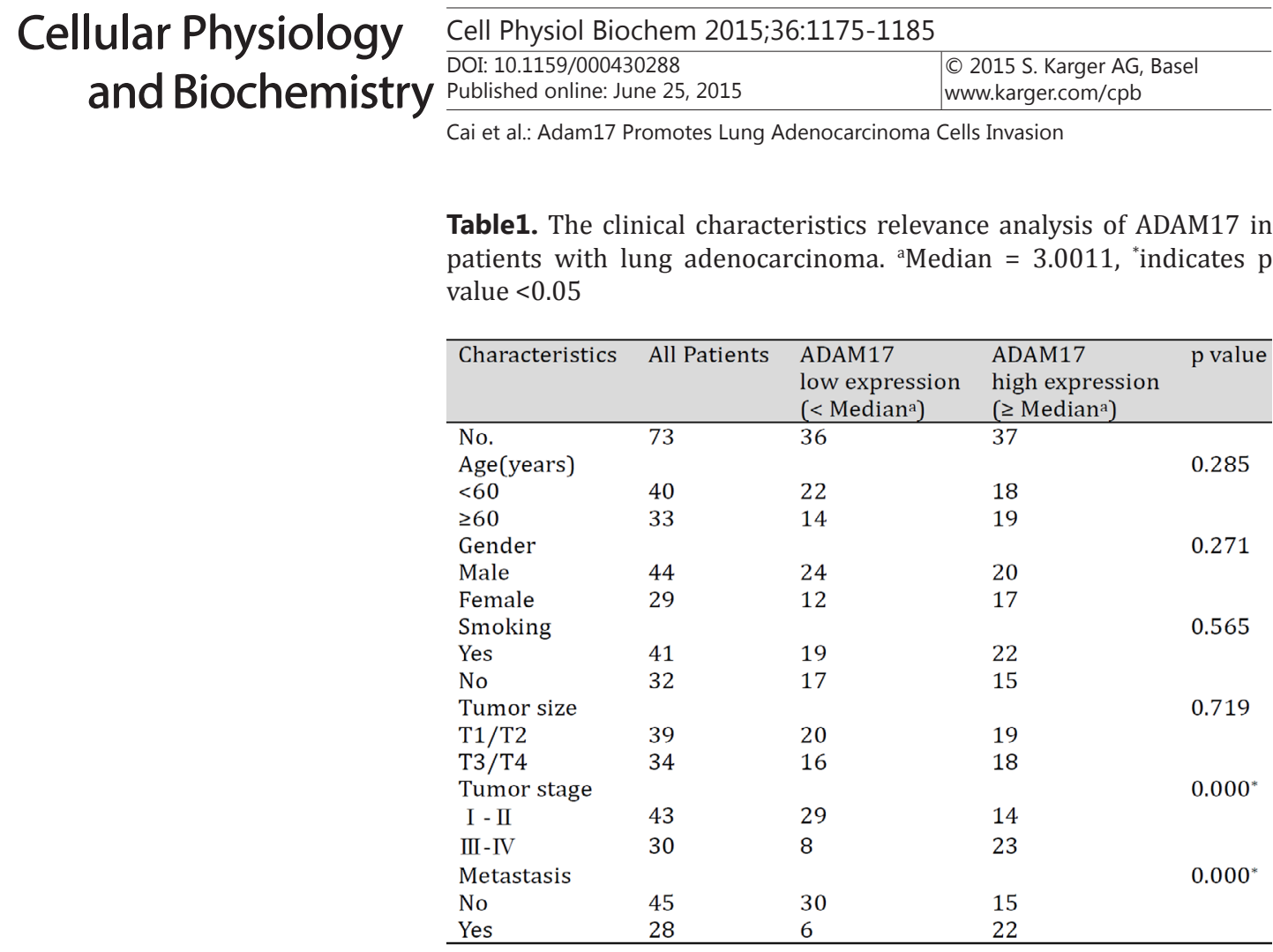

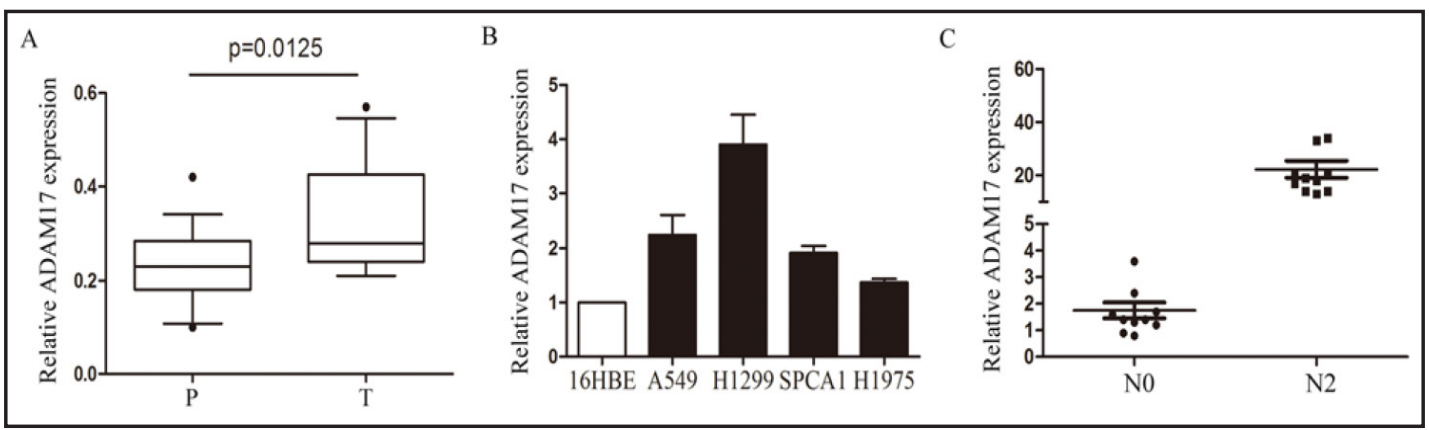

Fig. 1. ADAM17 was increased in lung adenocarcinoma tissues and cell lines. A: Box-plot analysis of ADAM17 mRNA level in 73 lung adenocarcinoma samples and corresponding adjacent tissues. B: ADAM17 mRNA level was detected in cell lines (A549, H1299, SPCA1 and H1975) and normal lung cell (16HBE). P refers to corresponding adjacent tissues and T refers to tumor tissues. C: ADAM17 expression level was investigated in 10 pairs of 2 stage (LN metastasis-positive) and N0 stage (LN metastasis negative) lung adenocarcinoma specimens by using qRT-PCR. Data are shown as mean \pm SEM of three independent experiments. * indicates significant difference compared with control group $(p<0.05)$.

analysis showed that ADAM17 mRNA levels were higher in the $\mathrm{N}_{2}$ group than in the $\mathrm{N}_{0}$ group $(\mathrm{p}<0.05$; Fig. 1C). Taken together, these results suggested that ADAM17 played a crucial role in the metastasis of lung adenocarcinoma.

Next, to clarify the role of ADAM17 in lung adenocarcinoma, we chose A549 and SPCA1 cell lines to perform a transwell invasion assay in vitro. ADAM17 expression was upregulated by transfecting with lentivirus, and down-regulated by siRNA. The transfection efficiencies were confirmed by western blot assay (Fig. 2A). Both A549 and SPCA1 cells invasive phenotype could be significantly enhanced when ADAM17 was overexpressed and evidently suppressed when ADAM17 was down-regulated (Fig. 2B). Together, these results suggested that ADAM17 promoted cells invasion in lung adenocarcinoma.

ADAM17 induced epithelial-to-mesenchymal transition (EMT)

Moreover, to determine if molecular changes typical of EMT occurred in cell lines, the expression of the epithelial marker (E-cadherin) and mesenchymal markers (including KARGER 


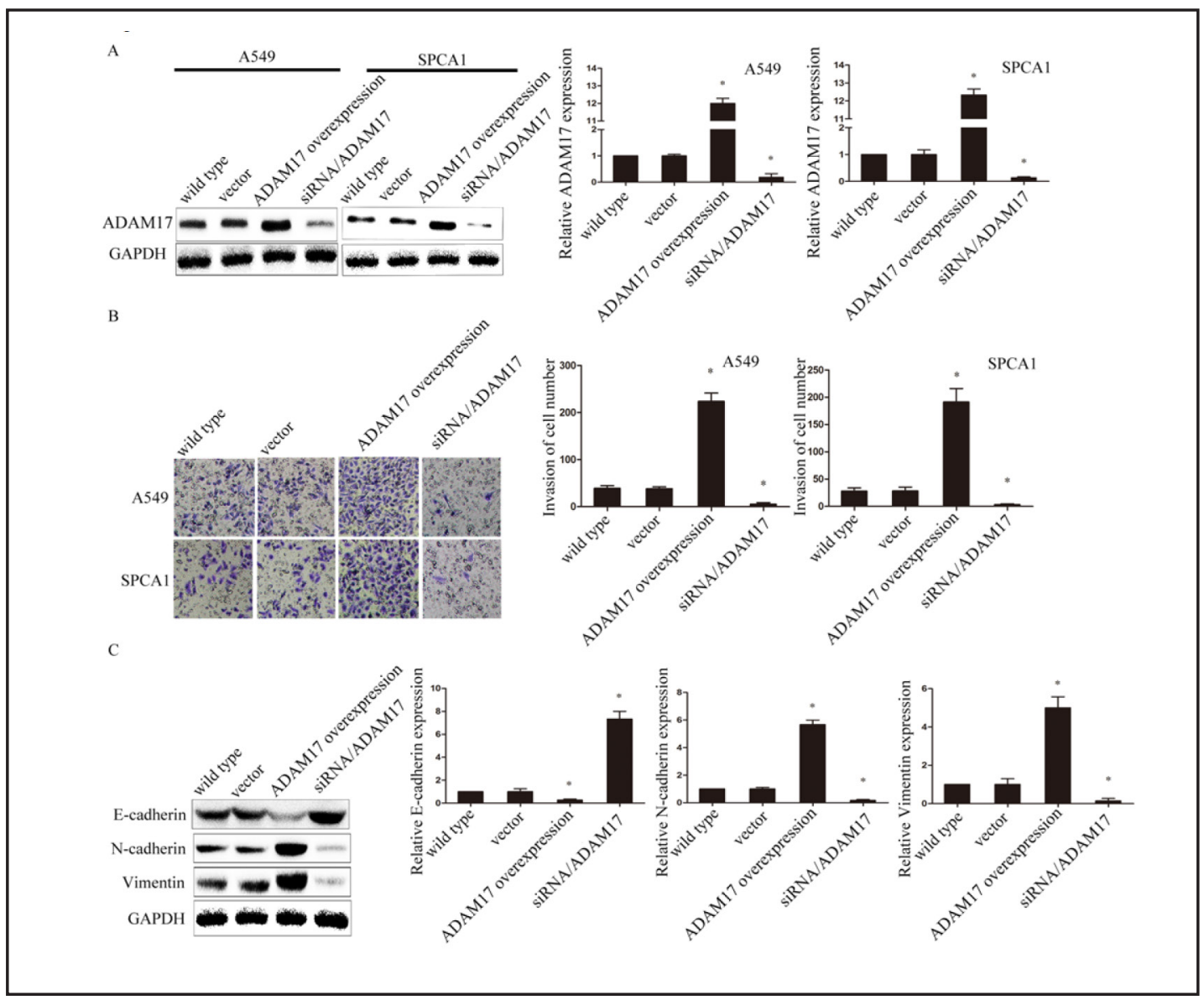

Fig. 2. ADAM17 promoted cells invasion and induced EMT. A: The results of ADAM17 expression in cell lines transfected with lentiviral vector (named ADAM17 overexpression) or siRNA (named siRNA/ ADAM17) were validated by using western blot assay. Cell lines (A549 and SPCA1) wild type and cells transfected with vector were used as control groups. B: Transwell assay was performed according to the Materials and Methods. The representative images of invasive cells at the bottom of the membrane stained with crystal violet were visualized as shown. C: During A549 cells, E-cadherin, N-cadherin and Vimentin protein expression levels were detected by using western blot assay. Data are shown as mean \pm SEM of three independent experiments. * indicates significant difference compared with control group ( $<<0.05)$.

N-cadherin and Vimentin) was examined in A549 cells by western blot assay. The assay showed that up-regulated ADAM17 expression resulted in decreased E-cadherin expression and evaluated N-cadherin and Vimentin expression. Meanwhile, suppressed ADAM17 expression resulted in increased E-cadherin expression and decreased $\mathrm{N}$-cadherin and Vimentin expression (Fig. 2C). This finding indicated that ADAM17 might contribute to regulating EMT marker expression in lung adenocarcinoma in vitro.

\section{ADAM17 was a direct target gene of miR-326}

Furthermore, based on the regulation pattern of ADAM17 in lung adenocarcinoma, we predicted that ADAM17 was the direct target gene of miR-326 by using bioinformatics analysis, microRNA.org (http://www.microrna.org/microrna/), miRDB (http://mirdb. org/cgi-bin/), and TargetScan (http://www.targetscan.org/) database. The bioinformatic software indicated that 3'-UTR (untranslated region) of ADAM17 binds to miR-326 with the high score. Thus, to further detect whether the expression level of ADAM17 expression regulated by miR-326 was due to the binding of miR-326 in the 3'-UTR of ADAM17, we cloned the 3'-UTR fragment containing the predicted site into pGL3 luciferase reporter 


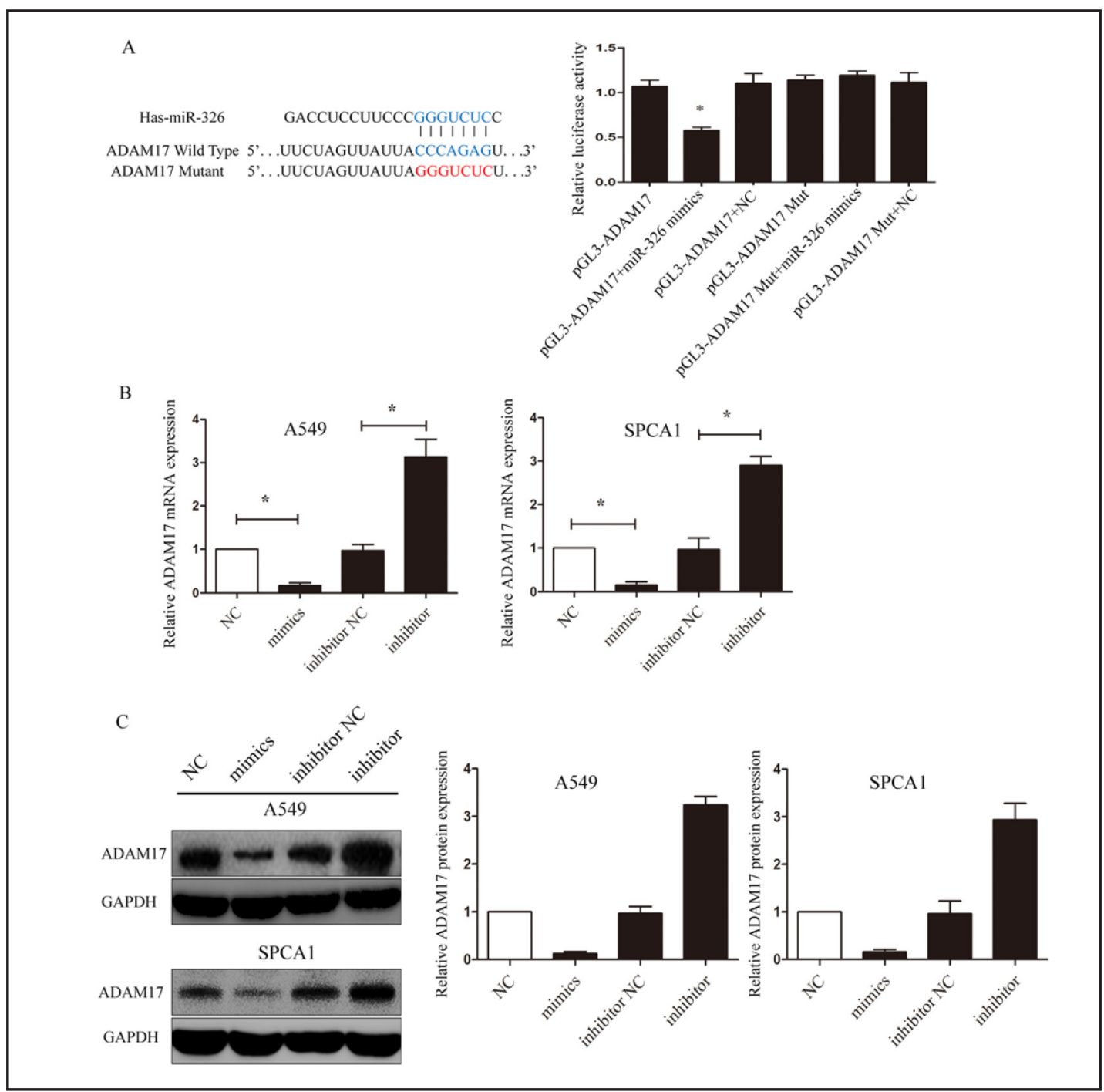

Fig. 3. miR-326 regulated ADAM17 by binding its 3'-UTR. A: The potential miR-326 seed region at the 3'UTR of ADAM17 mRNA was predicted by using bioinformatics analysis; A549 cells were co-transfected with miR-326 mimics (or NC) with pGL3-ADAM17 (or pGL3-ADAM17-Mut) vector. Luciferase activity was normalized by the ratio of firefly and Renilla luciferase signals. B and C: ADAM17 mRNA and protein expression levels in cells transfected with NC, miR-326 mimics, inhibitor NC and miR-326 inhibitor were detected by using qRT-PCR and western-blotting. GAPDH was regarded as an internal control. Data are shown as mean \pm SEM of three independent experiments. * indicates significant difference compared with control group (p $<0.05)$.

vector (pGL3-ADAM17) according to the results of prediction. In the predicted target site, the 3'-UTR fragment with mutant sequence was cloned as a control group (pGL3-ADAM17-Mut). Next, the miRNA luciferase reporter assay showed remarkable reduction activity in A549 cells with miR-326 mimics and pGL3-ADAM17 vectors as compared with the control and the mutant type (Fig. 3A). This result indicated that miR-326 targeted ADAM17. To further clarify ADAM17 expression level responses to the changes of miR-326, we performed qRTPCR and western blot assays. Both in mRNA and protein expression levels, up-regulation of miR-326 expression decreased ADAM17 expression; on the other hand, miR-326 inhibition increased ADAM17 expression level (Fig. 3B and C), suggesting that miR-326 could control ADAM17 expression by binding its 3'-UTR.

\section{KARGER}


Cai et al.: Adam17 Promotes Lung Adenocarcinoma Cells Invasion

Table 2. The clinical characteristics relevance analysis of miR-326 in patients with lung adenocarcinoma. ${ }^{\mathrm{b}}$ Median $=0.3017$, ${ }^{*}$ indicates $\mathrm{p}$ value $<0.05$

\begin{tabular}{|c|c|c|c|c|}
\hline Characteristics & All Patients & $\begin{array}{l}\text { miR-326 } \\
\text { low expression } \\
\left(<\text { Median }^{b}\right)\end{array}$ & $\begin{array}{l}\text { miR-326 } \\
\text { high expression } \\
\left(\geq \text { Median }^{b}\right)\end{array}$ & $\begin{array}{l}\mathrm{p} \\
\text { value }\end{array}$ \\
\hline No. & 73 & 37 & 36 & \multirow{4}{*}{0.417} \\
\hline Age(years) & & & & \\
\hline$<60$ & 40 & 22 & 18 & \\
\hline$\geq 60$ & 33 & 15 & 18 & \\
\hline Gender & & & & \multirow[t]{3}{*}{0.197} \\
\hline Male & 44 & 25 & 19 & \\
\hline Female & 29 & 12 & 17 & \\
\hline Smoking & & & & \multirow{3}{*}{0.401} \\
\hline Yes & 41 & 19 & 22 & \\
\hline No & 32 & 18 & 14 & \\
\hline Tumor size & & & & \multirow[t]{3}{*}{0.719} \\
\hline T1/T2 & 39 & 19 & 20 & \\
\hline T3/T4 & 34 & 18 & 16 & \\
\hline Tumor stage & & & & \multirow{3}{*}{$0.000^{*}$} \\
\hline I - II & 43 & 13 & 30 & \\
\hline III-IV & 30 & 24 & 6 & \\
\hline Metastasis & & & & \\
\hline No & 45 & 16 & 29 & \multirow[t]{2}{*}{$0.000^{*}$} \\
\hline Yes & 28 & 21 & 7 & \\
\hline
\end{tabular}

miR-326 inhibited lung adenocarcinoma cells invasion

Thus, we detected the expression levels of miR-326 by qRT-PCR in lung adenocarcinoma specimens $(n=73)$ as compared with corresponding adjacent tissues. Significantly, we discovered decreased expression levels of miR-326 in tumor tissues $(\mathrm{p}<0.05$; Fig. 4A). The aberrant expression of miR-326 was highly associated with tumor stage and metastasis (Table 2), suggesting that miR-326 played an important role in the metastasis of lung adenocarcinoma. Moreover, an inverse correlation was observed between miR-326 and ADAM17 ( $R=-0.846$; $p<0.0001$; Fig. 3B) in cancer samples, which was consistent with the results in vitro.

Furthermore, to investigate the underlying functions of miR-326 in lung adenocarcinoma, cell lines were transfected with NC (miR-control), miR-326 mimics, inhibitor NC (miR-326 inhibitor control) and miR-326 inhibitor respectively. The efficiency of transfection was examined by using qRT-PCR assay (Fig. 4C). Significantly, we found that up-regulation of miR326 inhibited cell lines invasion, on the other hand, down-regulation of miR-326 expression promoted the invasive ability of lung adenocarcinoma cell lines by using the transwell invasion assay (Fig. 4D). Meanwhile, the western blot assay showed that up-regulated miR326 expression resulted in increased E-cadherin expression and decreased $\mathrm{N}$-cadherin and Vimentin expression. Meanwhile, suppressed miR-326 expression resulted in decreased E-cadherin expression and evaluated N-cadherin and Vimentin expression (Fig. 4E). This result suggested that the abnormal expression level of miR-326 had the ability of regulating cell lines invasion and EMT.

Down-regulation of ADAM17 by siRNA reversed the effect of miR-326 inhibitor

To explore whether the functional effect of miR-326 was dependent on ADAM17, miR-326 inhibitor and siRNA/ADAM17 was co-transfected into cell lines. The transfection cells of miR-326 inhibitor and the co-transfection cells of miR-326 inhibitor and siRNA/ control were regarded as the control groups. The western blot assay was used to examine the transfection efficiency (Fig. 5A). Furthermore, the transwell invasion assay showed that down-regulation of ADAM17 partially abolished the enhancement of cells invasion induced by miR-326 inhibition, as shown in Fig. 5B. Meanwhile, the western blot assay showed that E-cadherin was increased and N-cadherin (Vimentin) was decreased in cells co-transfecting with miR-326 inhibitor and siRNA/ADAM17 as compared with the control groups (Fig. 5C). 


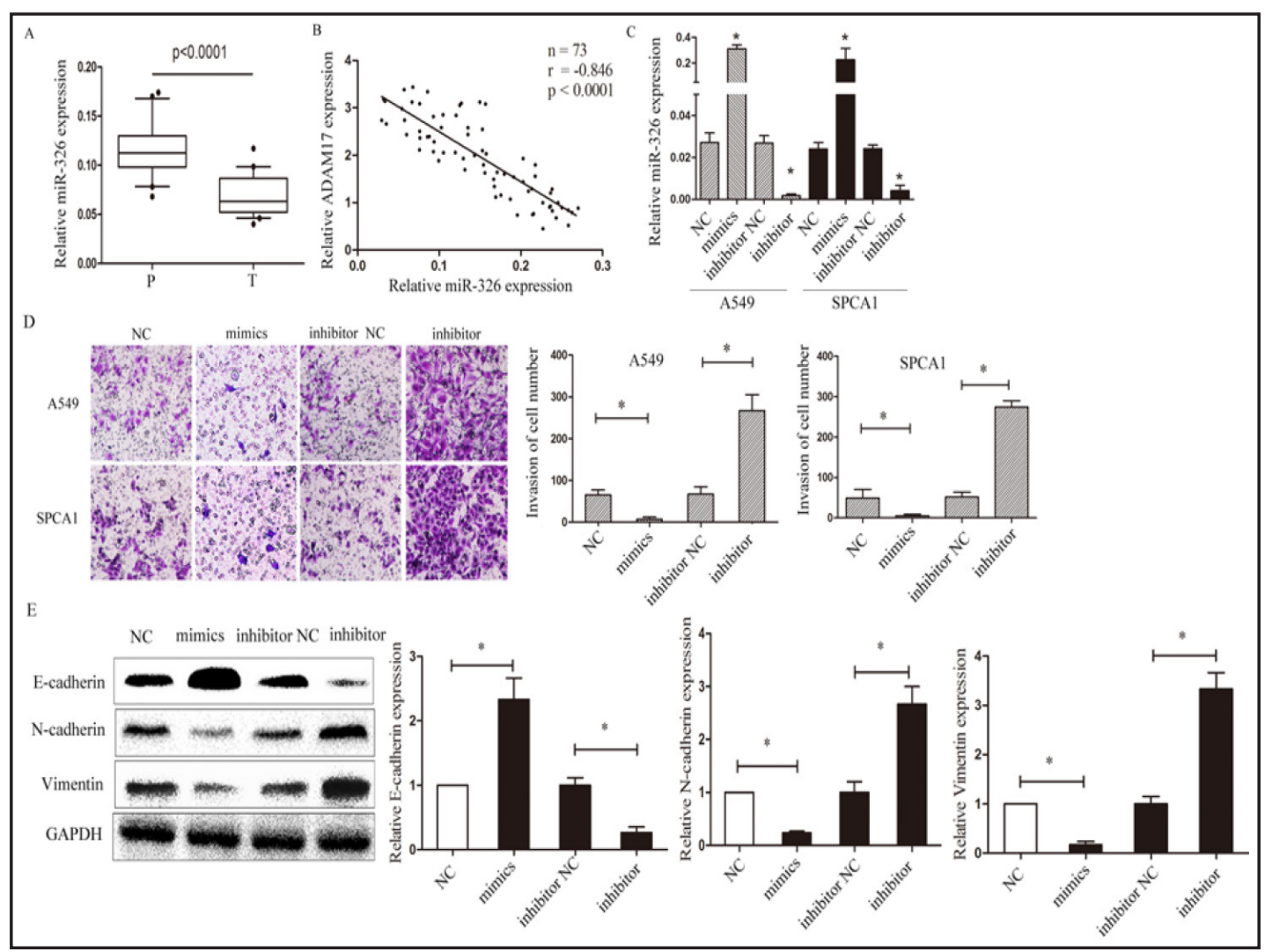

Fig. 4. Decreased expression of miR-326 promoted cells invasion and induced EMT in lung adenocarcinoma. A: Box-plot analysis of miR-326 level in 73 lung adenocarcinoma samples and corresponding adjacent tissues. B: A negative correlation was found between expression of miR-326 and ADAM17 in tumor samples $(\mathrm{R}=-0.846 ; \mathrm{p}<0.0001)$. C: The results of miR-326 expression in cell lines transfected with control for miR-mimics (NC), miR-326 mimics and control for miR-326 inhibitor (inhibitor NC), miR-326 inhibitor were validated by using qRT-PCR. D: Transwell assay was performed according to the Materials and Methods. A549 and SPCA1 were treated with NC, miR-326 mimics, inhibitor NC and miR-326 inhibitor for 24h. The representative images of invasive cells at the bottom of the membrane stained with crystal violet were visualized as shown. E: During A549 cells, E-cadherin, N-cadherin and Vimentin protein expression levels were detected by using western blot assay. Data are shown as mean \pm SEM of three independent experiments. * indicates significant difference compared with control group $(\mathrm{p}<0.05)$.

\section{Discussion}

Indeed, patients with lung cancer often exhibit tumor cell invasion and metastasis before diagnosis, which renders current treatments, including surgery, radiotherapy, and chemotherapy, ineffective. The underlying molecular mechanism of lung adenocarcinoma metastasis is still unclear. Therefore, studying the molecular basis of lung adenocarcinoma is crucial for designing new therapeutic agents that will improve the survival rate.

Evidence supporting the role of ADAM17 in cancer progression, invasion and metastasis development has been steadily accumulating $[13,14]$. All their findings supported the view that ADAM17 is an oncogene. Here, we found that ADAM17 was overexpressed in lung adenocarcinoma samples and associated with tumor stage and metastasis. In vitro, up-regulated ADAM17 expression promoted cells invasion, and down-regulated ADAM17 expression inhibited cells invasion. Baumgart et al. reported that ADAM17 regulates epidermal growth factor receptor expression through the activation of Notch1 in non-small 


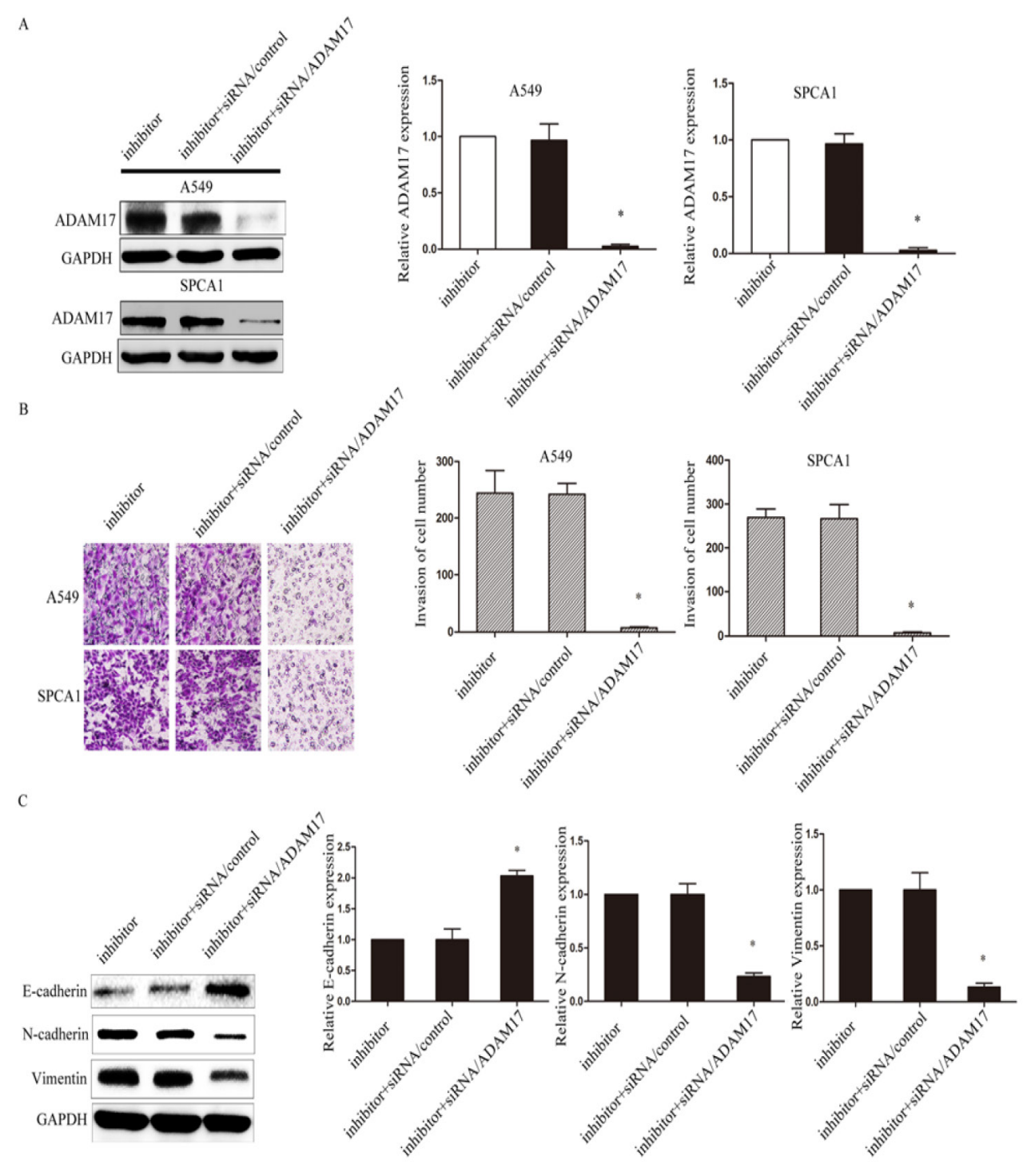

Fig. 5. Down-regulation of ADAM17 partially reverses the effect of miR-326 inhibition. A: The transfection efficiency was validated by using western-blotting after both A549 and SPCA1 cells transfected with miR-326 inhibitor and inhibitor+siRNA/ADAM17 (the co-transfection cells of miR-326 inhibitor and siRNA/ ADAM17) and inhibitor+siRNA/control (co-transfected with miR-326 inhibitor and siRNA/control). GAPDH was used as a control. Average values of integrated optical density (IOD) were assessed and recorded in the histogram. B: Transwell assay was performed as described in Materials and Methods. Cells were treated with miR-326 inhibitor and inhibitor+siRNA/ADAM17 (the co-transfection cells of miR-326 inhibitor and siRNA/ADAM17) and inhibitor+siRNA/control (co-transfected with miR-326 inhibitor and siRNA/control) for $24 \mathrm{~h}$. The representative images of invasive cells at the bottom of the membrane stained with crystal violet were visualized as shown. C: During A549 cells, E-cadherin, N-cadherin and Vimentin protein expression levels were detected by using western blot assay. Data are shown as mean \pm SEM of three independent experiments. * indicates significant difference compared with control group $(\mathrm{p}<0.05)$.

cell lung cancer [15]. Here, we found ADAM17 could regulate EMT in lung adenocarcinoma.

Epithelial-to-mesenchymal transition (EMT) contributes into cells invasion of many cancers. A significant breakdown of the tight junctions is involved in loss of epithelial marker (E-cadherin) and acquisition of mesenchymal makers ( $\mathrm{N}$-cadherin and Vimentin) 
[16-18]. In our study, we found that upregulated ADAM17 expression resulted in decreased E-cadherin expression and evaluated $\mathrm{N}$-cadherin and Vimentin expression, suppressed ADAM17 expression resulted in increased E-cadherin expression and decreased N-cadherin and Vimentin expression, which suggested that ADAM17 could affect EMT.

miRNAs bind target mRNAs at complementary sites in their 3'-untranslated regions (3'UTRs), thereby suppressing the expression of the target gene at the posttranscriptional level [19-21]. Through this mechanism, miRNAs regulate a wide range of biological processes, including cell proliferation and differentiation [22], migration, apoptosis, development, and metabolism [23-25]. For example, miR-545 suppress cell proliferation by targeting Cyclin D1 and CDK4 in lung cancer cells [26]. Here, the expression of miR-326 was significantly decreased in lung adenocarcinoma tissues and cell lines. Aberrant expression of miR-326 was related to the cells invasion and EMT. Furthermore, we also found miR-326 overexpression diminished but miR-326 knockdown increased ADAM17 expression level by binding the 3'UTR of ADAM17. Importantly, we confirmed that ADAM17, a target of miR-326, promoted EMT-induced cells invasion in lung adenocarcinoma.

In conclusion, we investigated that increased expression of ADAM17 in lung adenocarcinoma regulates mechanisms in the control of cells invasion. Up-regulation of ADAM17 induced EMT. Moreover, abnormal expression of miR-326 regulated cells invasion by binding ADAM17 3'-UTR. As the limit on the number of lung adenocarcinoma samples and cell types, more studies are necessary for further exploration of the potential role of ADAM17 in tumorigenesis.

\section{Abbreviations}

3'-UTR (3'-untranslated regions); qRT-PCR: (Quantitative Real Time- Polymerase Chain Reaction); FBS: (fetal calf serum); EDTA: (ethylene Diamine Tetraacetic Acid); EMT: (epithelial-to-mesenchymal transition).

\section{Acknowledgements}

We thank the teachers in our lab for supporting our work.

\section{Disclosure Statement}

There is no conflict among the authors.

\section{References}

1 Jemal A, Siegel R, Xu J, Ward E: Cancer statistics, 2010. CA Cancer J Clin 2010;60:277-300.

2 Aydin D, Bilici A, Yavuzer D, Kefeli U, Tan A, Ercelep O, Mert A, Yuksel S, Ozcelik M, Isik D, Surmeli H, Odabasi $\mathrm{H}$, Aliustaoglu M: Prognostic significance of adam17 expression in patients with gastric cancer who underwent curative gastrectomy. Clin Transl Oncol 2015, epub ahead of print.

3 Liu Y, Wu C, Wang Y, Wen S, Wang J, Chen Z, He Q, Feng D: Microrna-145 inhibits cell proliferation by directly targeting adam17 in hepatocellular carcinoma. Oncol Rep 2014;32:1923-1930.

4 Lv X, Li Y, Qian M, Ma C, Jing H, Wen Z, Qian D: Adam17 silencing suppresses the migration and invasion of non-small cell lung cancer. Mol Med Rep 2014;9:1935-1940.

$5 \quad$ Ni SS, Zhang J, Zhao WL, Dong XC, Wang JL: Adam17 is overexpressed in non-small cell lung cancer and its expression correlates with poor patient survival. Tumour Biol 2013;34:1813-1818. 


\section{Cellular Physiology Cell Physiol Biochem 2015;36:1175-1185 \begin{tabular}{l|l|l}
\cline { 2 - 2 } DOI: 10.1159/000430288 & C 2015 S. Karger AG, Basel
\end{tabular} and Biochemistry Published online: June 25, 2015

6 Cheng L, Yang T, Kuang Y, Kong B, Yu S, Shu H, Zhou H, Gu J: Microrna-23a promotes neuroblastoma cell metastasis by targeting cdh1. Oncol Lett 2014;7:839-845.

7 Rostas JW, 3rd, Pruitt HC, Metge BJ, Mitra A, Bailey SK, Bae S, Singh KP, Devine DJ, Dyess DL, Richards WO, Tucker JA, Shevde LA, Samant RS: Microrna-29 negatively regulates emt regulator n-myc interactor in breast cancer. Mol Cancer 2014;13:200.

8 Feng X, Wang Y, Ma Z, Yang R, Liang S, Zhang M, Song S, Li S, Liu G, Fan D, Gao S: Microrna-645, upregulated in human adencarcinoma of gastric esophageal junction, inhibits apoptosis by targeting tumor suppressor ifit2. BMC cancer 2014;14:633.

9 Wu N, Zhang C, Bai C, Han YP, Li Q: Mir-4782-3p inhibited non-small cell lung cancer growth via usp14. Cell Physiol Biochem 2014;33:457-467.

10 Wu L, Hui H, Wang LJ, Wang H, Liu QF, Han SX: Microrna-326 functions as a tumor suppressor in colorectal cancer by targeting the nin one binding protein. Oncol Rep 2015;33:2309-2318.

11 Valencia K, Martin-Fernandez M, Zandueta C, Ormazabal C, Martinez-Canarias S, Bandres E, de la Piedra C, Lecanda F: Mir-326 associates with biochemical markers of bone turnover in lung cancer bone metastasis. Bone 2013;52:532-539.

12 Wang R, Chen XF, Shu YQ: Prediction of non-small cell lung cancer metastasis-associated micrornas using bioinformatics. Am J Cancer Res 2015;5:32-51.

13 Li G, Forest F, Feng G, Gentil-Perret A, Peoc'h M, Cottier M, Mottet N: A novel marker adam17 for clear cell renal cell carcinomas: Implication for patients' prognosis. Urol Oncol 2014;32:1272-1276.

14 Beck Gooz M, Maldonado EN, Dang Y, Amria MY, Higashiyama S, Abboud HE, Lemasters JJ, Bell PD: Adam17 promotes proliferation of collecting duct kidney epithelial cells through erk activation and increased glycolysis in polycystic kidney disease. Am J Physiol Renal Physiol 2014;307:F551-559.

15 Baumgart A, Seidl S, Vlachou P, Michel L, Mitova N, Schatz N, Specht K, Koch I, Schuster T, Grundler R, Kremer M, Fend F, Siveke JT, Peschel C, Duyster J, Dechow T: Adam17 regulates epidermal growth factor receptor expression through the activation of notch1 in non-small cell lung cancer. Cancer Res 2010;70:5368-5378.

16 Dong H, Xie L, Tang C, Chen S, Liu Q Zhang Q Zheng W, Zheng Z, Zhang H: Snail1 correlates with patient outcomes in e-cadherin-preserved gastroesophageal junction adenocarcinoma. Clin translat Oncol 2014;16:783-791.

17 Liu Y, Li H, Feng J, Cui X, Huang W, Li Y, Su F, Liu Q Zhu J, Lv X, Chen J, Huang D, Yu F: Lin28 induces epithelial-to-mesenchymal transition and stemness via downregulation of let-7a in breast cancer cells. PloS one 2013;8:e83083.

18 Bao YX, Cao Q, Yang Y, Mao R, Xiao L, Zhang H, Zhao HR, Wen H: Expression and prognostic significance of golgiglycoprotein73 (gp73) with epithelial-mesenchymal transition (emt) related molecules in hepatocellular carcinoma (hcc). Diagn Pathol 2013;8:197.

19 Krol J, Loedige I, Filipowicz W: The widespread regulation of microrna biogenesis, function and decay. Nat Rev Gen 2010;11:597-610.

20 Calin GA, Croce CM: Microrna signatures in human cancers. Nat Rev Cancer 2006;6:857-866.

21 Iwagami Y, Eguchi H, Nagano H, Akita H, Hama N, Wada H, Kawamoto K, Kobayashi S, Tomokuni A, Tomimaru Y, Mori M, Doki Y: Mir-320c regulates gemcitabine-resistance in pancreatic cancer via smarcc1. Br J Cancer 2013;109:502-511.

22 Kang IH, Jeong BC, Hur SW, Choi H, Choi SH, Ryu JH, Hwang YC, Koh JT: Microrna-302a stimulates osteoblastic differentiation by repressing coup-tfii expression. J Cell Physiol 2015;230:911-921.

23 Price NL, Ramirez CM, Fernandez-Hernando C: Relevance of microrna in metabolic diseases. Crit Rev Clin Lab Sci 2014:1-16. 2014 Dec;51(6):305-20.

24 Xia Y, Chen Q, Zhong Z, Xu C, Wu C, Liu B, Chen Y: Down-regulation of mir-30c promotes the invasion of non-small cell lung cancer by targeting mta1. Cell Physiol Biochem 2013;32:476-485.

25 Zhang H, Zhao M, Lv Z, Zhang X, Qin X, Wang H, Wang S, Su J, Lv X, Liu H, Du W, Zhou W, Chen X, Fei K: Mir138 inhibits tumor growth through repression of ezh2 in non-small cell lung cancer. Cell Physiol Biochem 2013;31:56-65.

26 Du B, Wang Z, Zhang X, Feng S, Wang G, He J, Zhang B: Microrna-545 suppresses cell proliferation by targeting cyclin d1 and cdk4 in lung cancer cells. PLoS One 2014;9:e88022. 